\title{
Prediction of polymer composite material products using neural networks
}

\author{
A.V. Gaganov, O.A. Karaeva, A.M. Kudrin, A.V. Kretinin and A.A. Gurtovoy ${ }^{\text {a }}$ \\ Federal Government-financed Educational Institution of Higher Education, Voronezh State Technical University, \\ Moscovsky prospect 14, Voronezh, Russian Federation
}

\begin{abstract}
This article contains results of mathematical modeling of technological processes for manufacture of pre-production polymer composite prototypes having certain performance characteristics conducted with application of engineering analysis software using artificial neural networks. The first step was to evaluate the statistic importance of outside parameters which influence on materials' properties. The main task was to acquire the function of dependence of the mechanical properties from the outside factors.
\end{abstract}

Keywords: binder; analysis; modeling; control.

\section{Introduction}

The basic aim of the conducted works was a multiple-factor study of technological processes (TP) for manufacture of pre-production prototypes made from polymer-matrix composite materials (PCM) comprising a carbon reinforcement filler and a meltable matrix.

The problem was stated as a study of TP factors influence on the matrix material content, surface density, and mechanical properties of the prototypes.

The results presented in this article were obtained in the frame of the program for "Research and development of the automated technological process for polymer composite products manufacture exemplified by the IL-76MD-90A airplane engine nacelle doors" conducted by Voronezh Aircraft Production Association (VASO) OJSC. Applied scientific research (ASR) is carried out by Voronezh State Technical University (VSTU) in accordance with the federal target program "Research and development activities in priority areas of the Russian Federation scientific and technological complex for 2014 - 2020", in the field of "Transportation and space systems".

\section{The task}

Manufacturing technology for prepregs based on unidirectional carbon tapes and meltable matrixes is schematically presented in Fig. 1.

Manufacturing technology can be conventionally divided into several stages:

- Line preparation and auxiliary materials placement;

- Preparation and placement of the main materials: the reinforcement filler and the matrix;

- Impregnation process.

${ }^{\text {a }}$ Corresponding author : agurtovoy100@gmail.com 


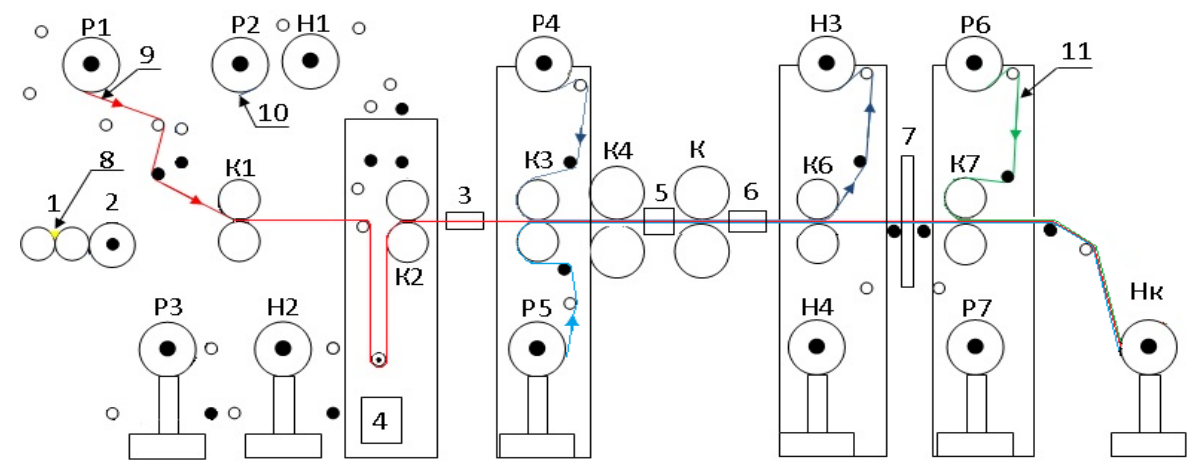

Figure 1. Schematic representation of the tape and auxiliary materials placement

1 - metering shafts, 2 - lamination shaft, 3 - IR furnace, 4 - matrix material bath, 5 - heating panel, 6 - cooling table, 7 - prepreg quality control system QMS-12 MAHLO, 8 - matrix material, 9 - cloth, 10 - paper, 11 - film, P1-P7 - unwinders, H1-H4 - winders, K1-K7 - calenders, $\bullet$ - strain sensors, $\bigcirc-$ auxiliary shafts or rollers

The prepreg is a unidirectional carbon tape using the Formosa $12 \mathrm{~K}$ carbon filament as a warp yarn and the VMPS-8 glass filament as a weft yarn. The tape is impregnated with the T-107 (and/or T-6815) adhesive (meltable) epoxy-based matrix at a given ratio by the method of matrix film application to a carbon tape.

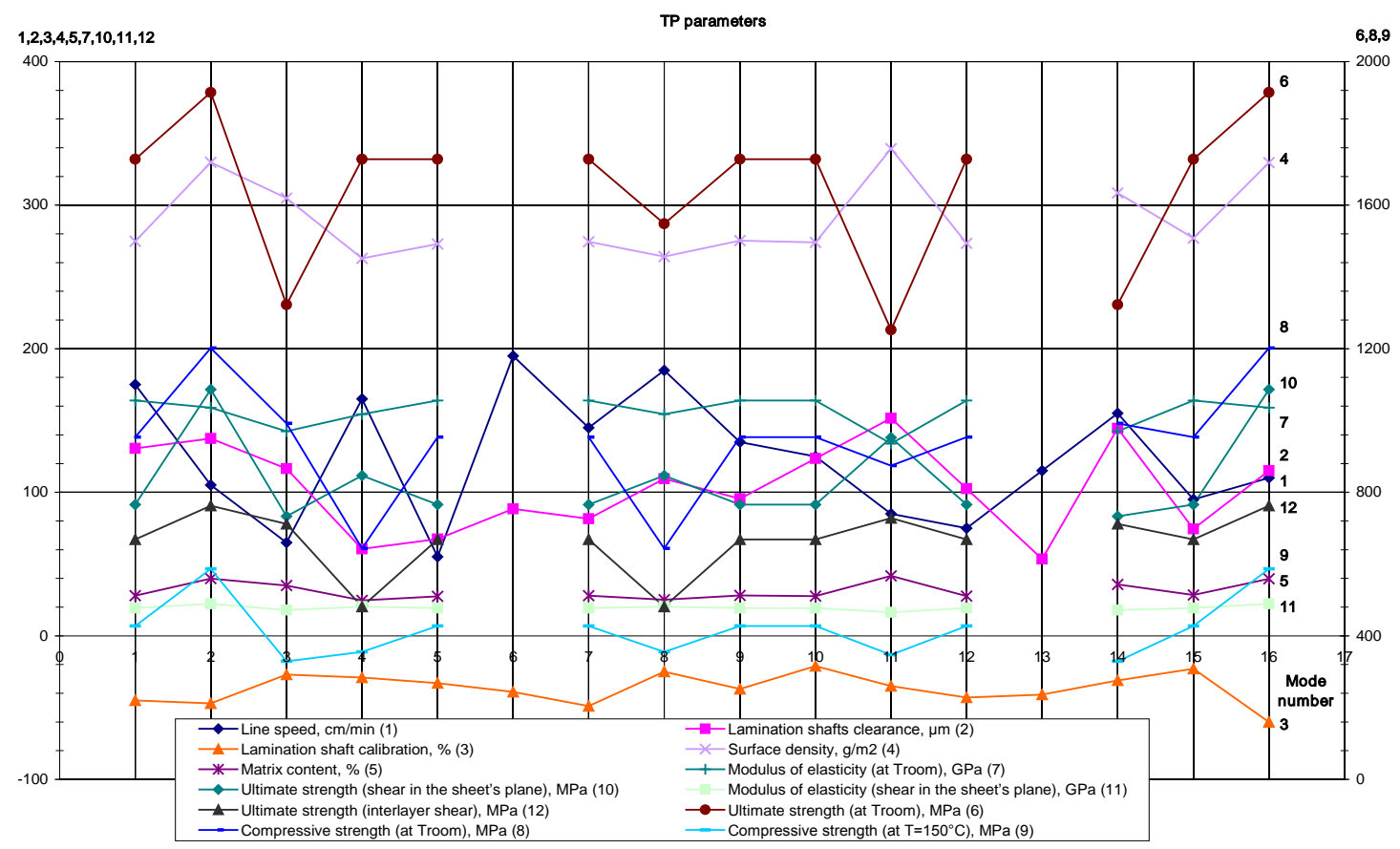

Figure 2. The results of TP execution

One of the practical objectives of this work was to create the tooling for automated control of carbon tape and meltable matrix TP factors providing maximum performance characteristics of the manufactured materials.

The input data used in solution of this task were material types and parameters, and operational characteristics of processes realized in the considered TP on VASO base.

At the experiment planning stage, the following variation ranges of the external factors were set: line speed $\mathrm{V}=0,5 \div 2 \mathrm{~m} / \mathrm{min}$, lamination shaft clearance $\mathrm{B} 2=50 \div 150 \mu \mathrm{m}$, calibration $\mathrm{K}=-50 \div-$ $20 \%$. 
The Optimal Space Filling Design experiment was planned with application of the DesignXplorer module for paramertic studies and non-linear optimization integrated into the ANSYS Workbench computer analysis engineering platform.

Matrix content in prepreg $(\mathrm{H})$ and surface densityR(o) were used as the quality criteria determined by results of experimental studies of hot melt prepreg manufacturing technology.

Fig. 2 shows schematic interpretation of the adhesive prepreg mechanical testing results obtained in different technological conditions taking into account the experiment plan.

Based on mechanical testing results, the following material strength characteristics were defined:

- Ultimate strength (at room temperature), SigR1;

- Modulus of elasticity (at room temperature), SigR2;

- Compressive strength (at room temperature), SigS1;

- Compressive strength (at $\mathrm{T}=150^{\circ} \mathrm{C}$ ), $\mathrm{SigS} 2$;

- Ultimate strength (shear in the sheet's plane), SigC1;

- Modulus of elasticity (shear in the sheet's plane), SigC2;

- Ultimate strength (interlayer shear), SigC3.

\section{Multi-factor analysis}

Multiple factor studies were conducted using the STATISTICA engineering system, including a mathematical module using neural network technology.

Neural networks are the universal approximation tool of the multivariate nonlinear dependences, capable "to be arranged" under the appearing of the new information of the researched process, i.e. they can serve as the intellectual tool of monitoring which is constantly filled up and clarified.

Fig. 3 presents the graphical notation of the computing artificial neural network (ANN) structure illustrating the process of intra-network computations.

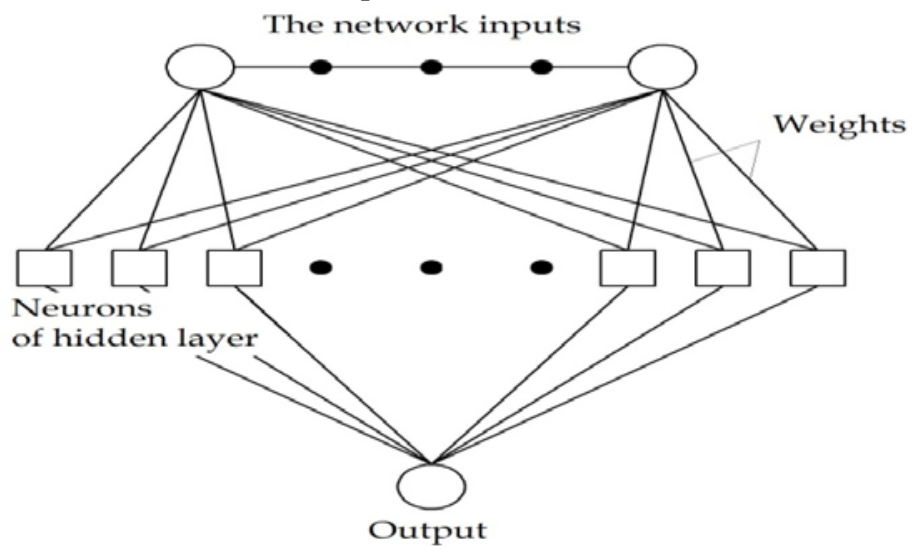

Figure 3. Neural network computing structure

To investigate the ANN approximated capabilities the perceptron with the single hidden layer (SLP) has been chosen as a basic model which performs nonlinear transformation of the input space into the output space in accordance with the formula (Bishop, 1995):

$$
y(\mathbf{w}, \mathbf{x})=\sum_{i=1}^{q} v_{i} f_{\sigma}\left(b_{i}+\sum_{j=1}^{n} w_{i j} x_{j}\right)+b_{0}
$$

where $\mathbf{x} \in \mathbf{R}^{n}$ - network input vector, made up of the values $x_{j} ; q$ - the neuron number of the single hidden layer; $\mathbf{w} \in \mathbf{R}^{s}$ - all weights and network thresholds vector; $w_{i j}$ - weight entering the 
model nonlinearly between $j$-m input and $i$-m neuron of the hidden layer; $v_{i}$ - output layer neuron weight corresponding to the $i$-neuron of the hidden layer; $b_{i}, b_{0}$-thresholds of neurons of the hidden layer and output neuron; $f_{\sigma}$ - activation function (in our case the logistic sigmoid is used). ANN of this structure already has the universal approximation capability, in other words it gives the opportunity to approximate the arbitrary analog function with any given accuracy. The main stage of using ANN for resolving of practical issues is the neural network model training, which is the process of the network weight iterative adjustment on the basis of the learning set (sample) $\left\{\mathbf{x}_{i}, y_{i}\right\}, \mathbf{x}_{i} \in \mathbf{R}^{n}, i=1, \ldots, k$ in order to minimize the network error - quality functional

$$
J(\mathbf{w})=\sum_{i=1}^{k} Q\left(f_{\varepsilon}(\mathbf{w}, i)\right)
$$

where $\mathbf{w}-$ ANN weight vector; $Q\left(f_{\varepsilon}(\mathbf{w}, i)\right)=f_{\varepsilon}(\mathbf{w}, i)^{2}-$ ANN quality criterion as per the $i$-training example; $f_{\varepsilon}(\mathbf{w}, i)=y\left(\mathbf{w}, \mathbf{x}_{i}\right)-y_{i}-i$-example error. For training purposes the statistically distributed approximation algorithms may be used based on the back error propagation or the numerical methods of the differentiable function optimization.

To solve the tasks of ANN training, it is important to attain good generalizing properties of the network, i.e. its capability to predict the values, which do not belong to the training sample. Thus, at the stage of training ANN, having a fixed structure, the problem arises in evaluating a certain functional of ANN performance, which is generally represented as the total quadratic error for a specified training sample, and the degree of correspondence to some subjective prior information about the type of the neuron-network response surface. This determines the necessity for regularization of fixed-structured ANN training.

Fig. 4 shows the representative results of the $H$ criterion behavior modeling obtained with the help of the built neural network models. For comparison, the same graph shows initial values of the criterion obtained according to the experiment results.

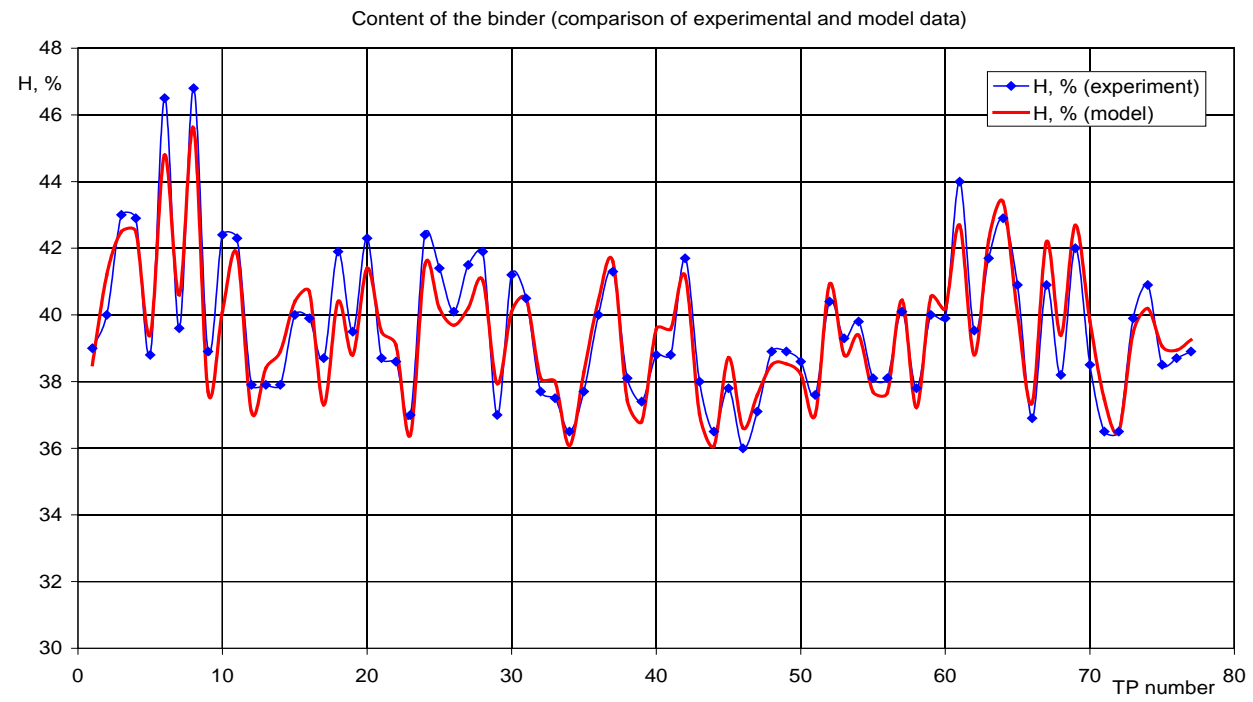

Figure 4. Neural network modeling results

The model's sensitivity to different factors was analyzed with the aid of the DesignXplorer module.

At the first stage, statistical significance of external conditions for material properties was estimated with application of dispersion analysis based on Pareto chart. It was found out that V and B2 factors are the most statistically significant for all parameters. The significant parameters were 
then used for building of the response surface charts and contour charts with the surface level lines marked on them.

Typical charts using SigS1 parameter as an example are shown in Fig. 5-7.

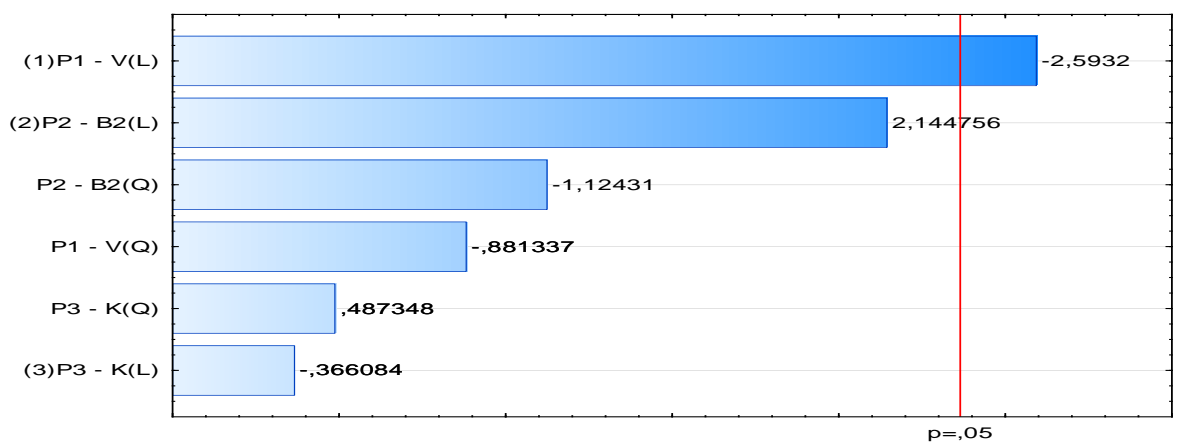

Figure 5. Pareto chart for SigS1

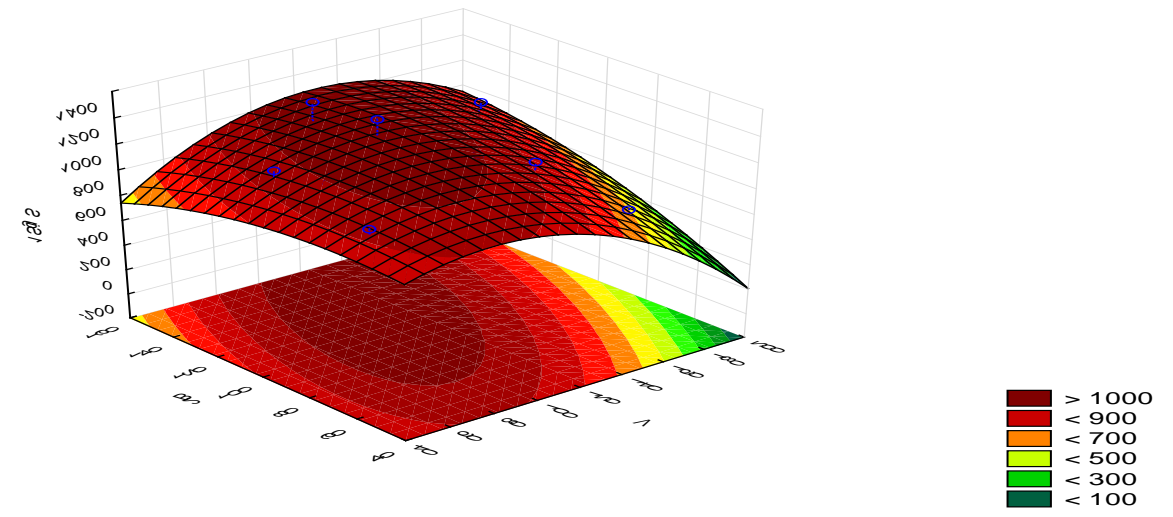

Figure 6. Response surface chart for SigS1

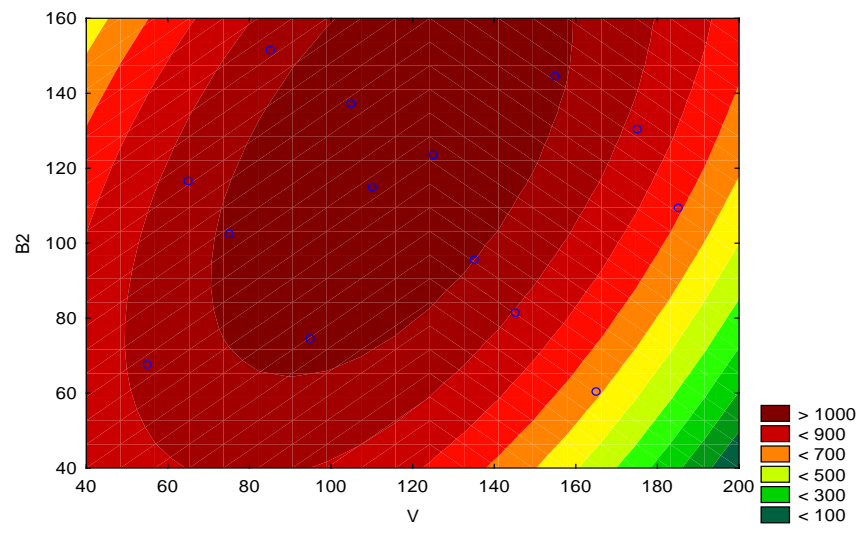

Figure 7. Surface level lines for SigS1

The main task was to establish dependencies of the mechanical properties on the external factors using procedures of multidimensional regression based on the full quadratic polynomial. Table 1 contains determination coefficients of the obtained multiple factor regression models. 
Table 1. Determination coefficients of regression models

\begin{tabular}{|c|c|c|c|c|c|c|c|}
\hline Property & Sig_R1 & Sig_R2 & Sig_S1 & Sig_S2 & Sig_C1 & Sig_C2 & Sig_C3 \\
\hline $\mathrm{R}^{2}$ & 0,786 & 0,734 & 0,823 & 0,767 & 0,887 & 0,805 & 0,983 \\
\hline
\end{tabular}

Multiple regression results provided a base for definition of mathematical dependencies that allow prediction of the material's mechanical properties:

$\operatorname{SigR} 1=1654,689-211,458 * \mathrm{~V}+27,252 * \mathrm{~B} 2-137,537 * \mathrm{~K}-447,135 * \mathrm{~V}^{\wedge} 2-$

$16,722 * \mathrm{~B} 2 \wedge 2+563,955 * \mathrm{~K}^{\wedge} 2+425,999 * \mathrm{~V} * \mathrm{~B} 2+413,836 * \mathrm{~V} * \mathrm{~K}-890,851 * \mathrm{~B} 2 * \mathrm{~K}$.

SigR $2=159,7009-18,7264 * \mathrm{~V}+10,6514 * \mathrm{~B} 2-5,7387 * \mathrm{~K}-33,3873 * \mathrm{~V}^{\wedge} 2-$

$4,5060 * \mathrm{~B} 2 \wedge 2+41,9386 * \mathrm{~K}^{\wedge} 2+29,3424 * \mathrm{~V} * \mathrm{~B} 2+34,1372 * \mathrm{~V} * \mathrm{~K}-70,9805 * \mathrm{~B} 2 * \mathrm{~K}$.

$\mathrm{SigS} 1=988,697-302,234 * \mathrm{~V}+308,779 * \mathrm{~B} 2-45,646 * \mathrm{~K}-397,782 * \mathrm{~V}^{\wedge} 2-$

$355,211 * \mathrm{~B} 2 \wedge 2+73,161 * \mathrm{~K}^{\wedge} 2+288,604 * \mathrm{~V} * \mathrm{~B} 2-158,280 * \mathrm{~V} * \mathrm{~K}-292,151 * \mathrm{~B} 2 * \mathrm{~K}$.

$\operatorname{SigS} 2=408,192-82,866 * \mathrm{~V}+42,877 * \mathrm{~B} 2-45,940 * \mathrm{~K}-128,903 * \mathrm{~V}^{\wedge} 2-$

$143,277 * \mathrm{~B} 2{ }^{\wedge} 2+141,709 * \mathrm{~K}^{\wedge} 2+96,407 * \mathrm{~V}^{*} \mathrm{~B} 2+84,380 * \mathrm{~V} * \mathrm{~K}-236,965 * \mathrm{~B} 2 * \mathrm{~K}$.

$\mathrm{SigC} 1=93,7963-35,8558 * \mathrm{~V}+53,4977 * \mathrm{~B} 2-59,9834 * \mathrm{~K}+6,5937 * \mathrm{~V}^{\wedge} 2+$

$35,3655 * \mathrm{~B} 2 \wedge 2+55,6043 * \mathrm{~K}^{\wedge} 2-39,5631 * \mathrm{~V} * \mathrm{~B} 2+74,7243 * \mathrm{~V} * \mathrm{~K}-86,6989 * \mathrm{~B} 2 * \mathrm{~K}$.

$\operatorname{SigC} 2=19,96278-0,26587 * \mathrm{~V}+0,16148 * \mathrm{~B} 2-2,2236 * \mathrm{~K}-1,11729 * \mathrm{~V}^{\wedge} 2-$

$0,25707 * \mathrm{~B} 2 \wedge 2+1,73786 * \mathrm{~K}^{\wedge} 2+0,87253 * \mathrm{~V} * \mathrm{~B} 2+3,85354 * \mathrm{~V} * \mathrm{~K}-5,44637 * \mathrm{~B} 2 * \mathrm{~K}$.

$\mathrm{SigC} 3=75,6566-21,9163 * \mathrm{~V}+29,3265 * \mathrm{~B} 2-22,9902 * \mathrm{~K}-$

$47,4452 * \mathrm{~V}^{\wedge} 2+5,2717 * \mathrm{~B} 2 \wedge 2+4,1305 * \mathrm{~K}^{\wedge} 2+27,4317 * \mathrm{~V} * \mathrm{~B} 2-39,112 * \mathrm{~V} * \mathrm{~K}+3,6708 * \mathrm{~B} 2 * \mathrm{~K}$.

Practically all dependencies have extremums in the considered area of the factor space.

Then, using the DesignXplorer module, sensitivity coefficients at average parameter values were calculated (Fig. 8).

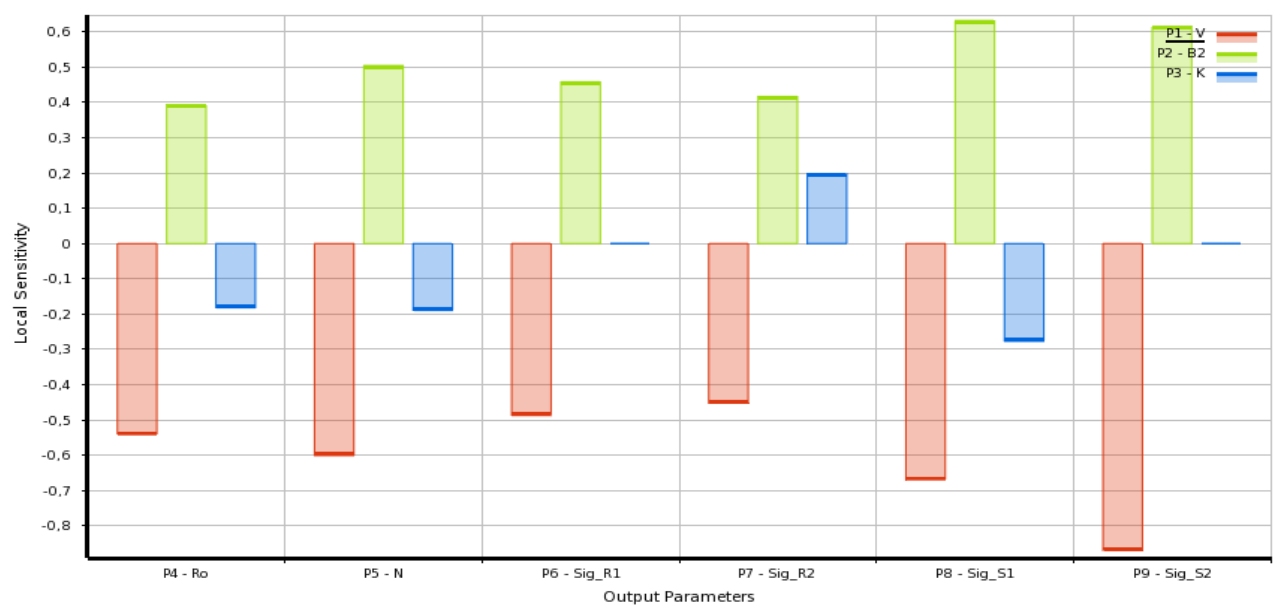

Figure 8. Calculated coefficients of sensitivity at average parameter values

It was found out that $\rho, \mathrm{H}$, Sig_R2 and Sig_S1 criteria at the average parameter values are sensitive to variation of the external conditions V, B2, K; Sig_R1 and Sig_S2 criteria - to variation of $\mathrm{V}, \mathrm{B} 2$ parameters.

Typical results of analysis for the $\rho$ criterion are presented in Fig. 9. 

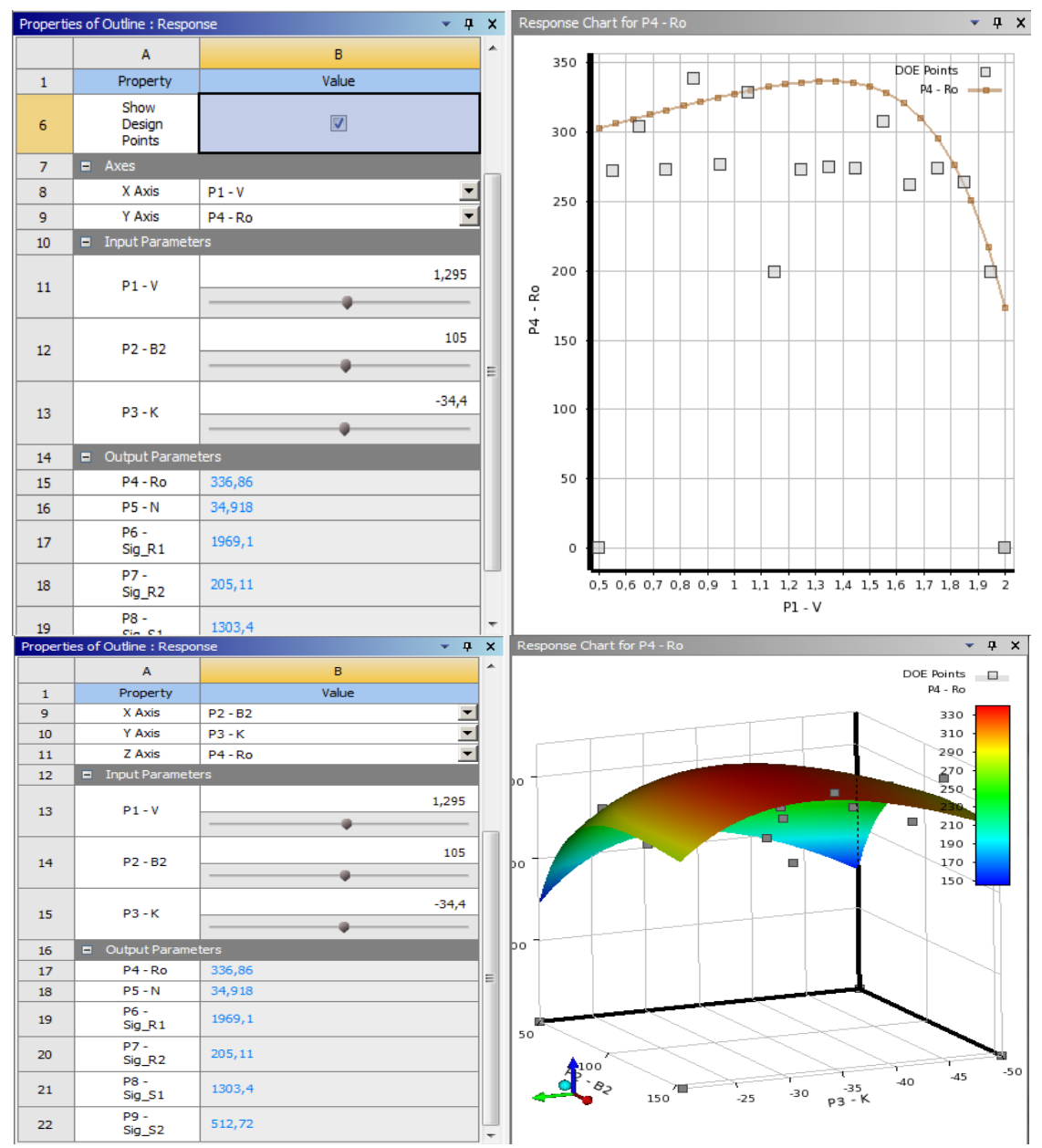

Figure 9. Dependencies obtained for the $\rho$ criterion
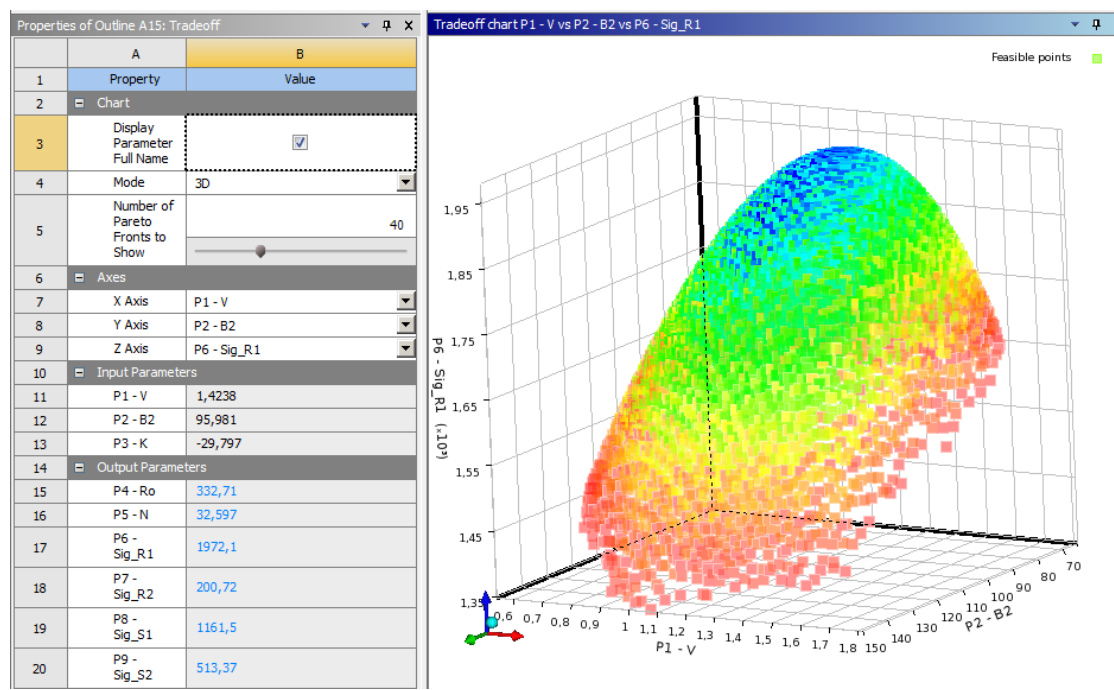

Figure 10. Results of multicriteria optimization 
The presence of extremums in the considered area of the factor space became a reason for optimization studies, including those in multicriteria statement.

Optimal values of input parameters V, B2 and $\mathrm{K}$ were defined with the aid of the DesignXplorer module.

Sig_R1, Sig_R2, Sig_S1 and Sig_S2 characteristics were used as the optimized criteria.

Representative results and some Pareto-optimal points of the factor space are shown in Fig. 10 (in process of optimization, the Screening method was used with analysis settings for 10000 points and selection of the three best results).

\section{Summary}

This paper contains results of the multiple factor study and mathematical modeling of technological processes for manufacture of pre-production polymer composite prototypes having the specified performance characteristics. The study and modeling have been conducted applying specialized software for parametric studies and non-linear optimization using artificial neural networks.

The algorithm of combined back propagation, as part of the concept of fixed-structured ANN training for obtaining the response surfaces of minimal curvature, and for enhancing the robust properties of the developed ANN of optimal structure that make it possible to approximate the arbitrary continuous function with any determination coefficients desired.

During the research, we have found which of the outside parameters have the largest impact on the results of these TP. The mathematical models were designed to forecast the results of the TP, which are now extremely unpredictable and stochastic.

\section{Acknowledgement}

The work was supported by the Ministry of Education and Science of Russian Federation in the frame work of Federal Special-Purpose Program (Agreement N 14.574.21.0083, unique identifier RFMEFI57414X0083).

\section{References}

1. A.V. Kretinin. "Method of Weighted Residuals on the Base of Neuronet;s Approximations for Computer Simulation of Hydrodynamics Problems", 2008 IEEE International Conference on Computational Cybernetics, 11/2008.

2. Valyuhov, Sergey, Alexander Kretinin, and Alexander Burakov. "Neural Network Modeling of Hydrodynamics Processes", Hydrodynamics - Optimizing Methods and Tools, (2011).

3. Design of fiber reinforced laminates for maximum fatigue life. AH Ertas, FO Sonmez. Procedia Engineering 2(1), 251-256.

4. Optimization of fiber-reinforced laminates for a maximum fatigue life by using the particle swarm optimization. Part II. AH Ertas. Mechanics of Composite Materials 49(1), 107-116.

5. Optimization of fiber-reinforced laminates for a maximum fatigue life by using the particle swarm optimization. Part I. AH Ertas. Mechanics of Composite Materials 48(6), 705-716.

6. Design optimization of fiber-reinforced laminates for maximum fatigue life. AH Ertas, FO Sonmez. Journal of Composite Materials. 